\title{
Color Doppler sonography of the aortic isthmus in intrauterine growth-restricted fetuses and normal fetuses
}

\author{
Ladan Younesi (1), Mostafa Ghadamzadeh (2), Ghazaleh Amjad (1), Zeinab Safarpour Lima (1)
}

(1) Shahid Akbar Abadi Clinical Research Development Unit (ShACRDU), Iran University of Medical Sciences (IUMS), Tehran, Iran; (2) Department of Radiology, Hasheminejad Kidney Center (HKC), Iran University of Medical Sciences, Tehran, Iran

This article is distributed under the terms of the Creative Commons Attribution Noncommercial License (CC BY-NC 4.0) which permits any noncommercial use, distribution, and reproduction in any medium, provided the original author(s) and source are credited.

\begin{abstract}
Intrauterine growth restriction is associated with a significant increase in morbidity and perinatal mortality, and increases the likelihood of fetal death, asphyxia, meconium aspiration, hypoglycemia, and neonatal hypothermia. The aim of this study was to determine aortic isthmus flow difference by using color doppler sonography in Intrauterine growth restriction and normal fetuses. The data presented were obtained from 30 mothers, who referred to the radiology department of Akbarabadi Hospital of Tehran with a diagnosis of intrauterine growth restriction. An ultrasound was performed to determine the status of placenta, fetus, and amniotic fluid. The umbilical arterial doppler assessment was used to confirm diagnosis of intrauterine growth restriction. Thirteen $(43.3 \%)$ were nulliparous mothers and $17(56.7 \%)$ were multiparous mothers. 30 pregnant women with healthy fetuses were enrolled as control group. According to the ultrasound findings, Dactus Venus wave type was recorded in intrauterine growth restriction fetuses, which was reported as normal (26 subjects; $86.7 \%)$ and abnormal (4 subjects; 13.3\%). All together, this study provides appropriate guidance to use doppler for delivery timing and to control risk factors.
\end{abstract}

Key Words: Color Doppler ultrasound, fetal aortic isthmus, Doppler flow, Intrauterine growth restriction.

Eur J Transl Myol 28 (4): 370-375, 2018

Intrauterine growth restriction (IUGR) is associated with a significant increase in morbidity and perinatal mortality, ${ }^{1,2}$ and the likelihood of some emerging risks and problems such as fetal death, birth asphyxia, meconium aspiration, hypoglycemia, neonatal hypothermia, and abnormal development of nervous system can be increased. ${ }^{3,4}$ Identifying fetuses with inappropriate growth is still difficult. Growth failure case identification is sometimes not possible even in the neonatal nursing unit, that shows this difficulty more than ever. However, there are clinical methods and more sophisticated technologies that help to diagnose maternal constraint of fetal growth. ${ }^{5-7}$ Determining the serial fundal heiht measurement is a safe, and relatively accurate screening method for the diagnosis of a large number of small fetuses for gestational age. ${ }^{8-10}$ However, the main disadvantage of this method is its low inaccuracy. In several studies, it was found that symphysial fundal height measurement (SFH) detects only $40 \%$ of these cases. Therefore, small fetuses for gestational age are both neglected and over-detected. In spite of this, these results do not reduce the importance of SFH as a simple screening method. ${ }^{11-13}$ The abnormality of fetal umbilical artery flow velocity has a unique association with IUGR. The abnormal result of this test is the absence or reduction of diastolic blood flow, which implies an increase in impedance. The use of Doppler velocity measurement has been suggested as a contributing method to other fetus assessments. ${ }^{14}$ It is worth noting that the aortic isthmus is the part of aorta located between the origin of the left subclavian artery and the ductus arteriosus. The timely and accurate diagnosis of IUGR cases leads to the necessary measures for the health of the fetus and the mother. Therefore, alternative methods for suspected cases of IUGR help to manage maternal and fetal statuses and provide access to appropriate diagnostic and therapeutic outcomes. Due to the different results of aortic isthmus from IUGR fetuses and lack of research in Iran, the present study was performed to evaluate color Doppler sonography of the aortic isthmus in normally grown and growth-restricted fetuses. 


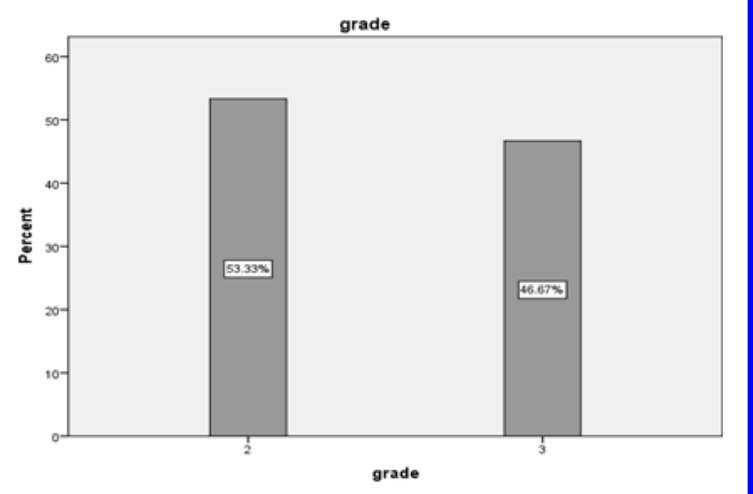

Fig 1. Percentage frequency of placental grading in IUGR fetuses.

\section{Materials and Methods}

This analytical cross-sectional study was conducted among mothers who referred to the radiology department of Akbar Abadi Hospital in Tehran during 2013-2014 with a diagnosis of IUGR from 25 to 37 weeks of gestation. The study was explained to enrolled persons and an informed consent was obtained before analyses. Standard sonography was performed to determine the status of placenta, fetus and amniotic fluid. Umbilical arterial Doppler assessment was used for definitive diagnosis of IUGR. Doppler evaluation of fetal aortic isthmus blood flow was also performed. In the prenatal care initial diagnosis of IUGR was performed on the basis of clinical examination and fundal height measurement. For this purpose, the area of the abdominal arc was measured using a tape (in $\mathrm{cm}$ ) from the top of the symphysis pubis to the uterine fundus with an empty bladder. Then, the percentile of the fetus was calculated, which was confirmed if it was below the 10th percentile. Pregnant mothers who did not have risk factors for IUGR and referred to the clinic of Akbar Abadi Hospital for prenatal care were included as control group. These mothers were evaluated by clinical examination, weight assessment, umbilical arterial Doppler assessment, as well as Doppler test of fetal aortic isthmus blood flow. Patient information such as fetal age, pregnancy status, parity and maternal age were also recorded in a data collection form. Doppler findings of the fetal aortic isthmus blood flow were determined in IUGR and healthy fetuses. The data of all the subjects who participated in this study remained strictly confidential. The analyses did not impose health system related costs to the subjects.

\section{Sample size}

Regarding the small number of available samples, we tried to use a census sampling for all subjects that were referred to this center during one year. Sample size included all patients diagnosed with IUGR in the Department of Radiology, Akbar Abadi Hospital.

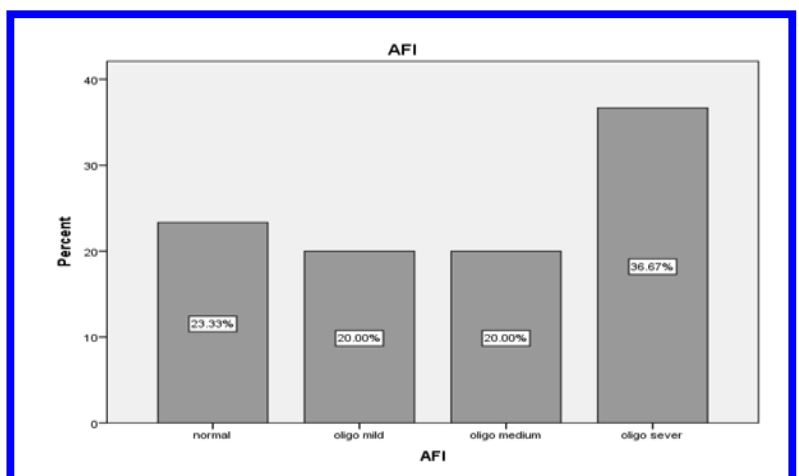

Fig 2. Amniotic fluid index in IUGR fetuses

Case group consisted of 30 subjects; 30 healthy subjects were included in the study as control group with healthy fetuses.

\section{Data analysis}

Data analysis was performed using IBM SPSS 22 software. Descriptive analysis was presented as mean, standard deviation and frequency percentage for underlying variables. Statistical analyses were performed using T-test and chi square tests. Values of $\mathrm{P}$ $<0.05$ were considered to be statistically significant.

\section{Results}

In this study, 30 pregnant women who referred to the radiology department of Akbarabadi Hospital in Tehran at 34 and 36 weeks of gestation were diagnosed with IUGR and 30 pregnant women with healthy fetuses were enrolled as control group. In the case group, the mean gestational age was $32.4(\mathrm{SD}=3.2)$ weeks. The minimum and maximum gestational age were determined to be 27 weeks and 37 weeks, respectively . Thirteen $(43.3 \%)$ were nulliparous mothers and 17 $(56.7 \%)$ were multiparous mothers. For all mothers, standard ultrasonography was performed to evaluate the placenta, fetal weight, and IUGR. Sixteen mothers of the case group $(53.3 \%)$ showed grade II placenta, followed by grade III placenta $(46.7 \%)$ (Figure 1). Amniotic fluid index was normal in $7(23.3 \%)$ cases, followed by 6 cases of mild oligohydramnios (20\%), 6 cases of moderate oligohydramnios $(20 \%)$ and 11 cases of severe oligohydramnios $(36.7 \%)$, (Figure 2). The fetal weight was recorded in 19 cases $(63.3 \%)$ of mothers (with IUGR fetuses) to be below the 3rd percentile, while 11 cases $(36.7 \%)$ were below the 10 th percentile, so the diagnosis of IUGR was confirmed in all cases. According to the ultrasound findings of the, the Dactus Venus wave type (DV) was recorded in IUGR fetuses, which was reported as normal (26 subjects; $86.7 \%$ ) and abnormal (4 subjects; $13.3 \%$ ), (Fig. 3). In the IUGR fetuses, the mean RI of the umbilical artery was $0.86(\mathrm{SD}=0.09)$, where the lowest and highest were recorded as 0.68 and 1 , all items were 


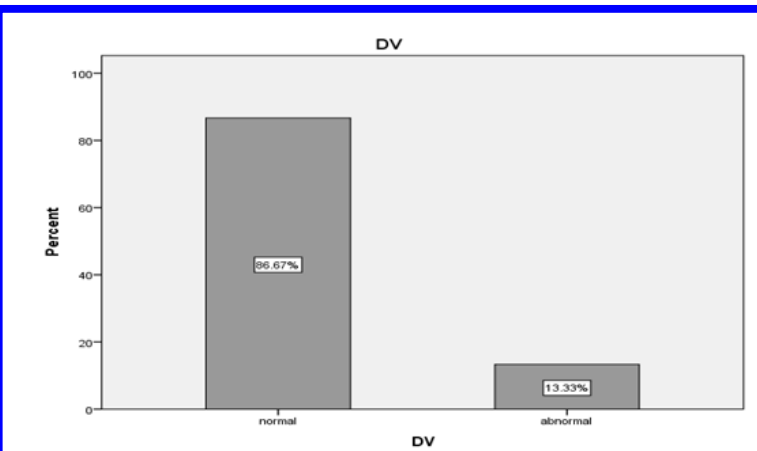

Fig 3. Percent doppler flow pattern in the ductus venosus (IUGR fetuses)

above the 95th percentile. In IUGR fetuses, the mean RI of the fetal aortic isthmus was calculated as 0.89 ( $\mathrm{SD}=$ $0.02)$, with the lowest and highest rate $(0.82$ and 0.94 ; fig 4). Furthermore, the mean RI of the fetal aortic isthmus in the control group was calculated as 0.87 (SD $=0.04)$, comparing the highest with the lowest scores (0.78 and 0.94; Table 1). Based on the one sample K-S test, the distribution of RI of the fetal aortic isthmus was non normal distribution. As a result, nonparametric test was employed for comparing the two groups. Based on the results of Mann-Whitney test, there was no significant difference in the mean of RI in the case and control group ( $\mathrm{P}=0.08)$, (Fig. 5).

\section{Discussion}

IUGR occurs in $15 \%$ of the second pregnancy due to utero-placental failure and is one of the major cause of fetal and neonatal morbidity and long-term health problems. ${ }^{16,17}$ This is a progressive vascular disorder associated with vascular malformations of the third villi that initiates hemodynamic disorders and abnormalities in multiple fetusess vessels. In the absence of effective intrauterine therapies, the timing of delivery and the birth of a baby become crucial. The main goal of controlling the delivery of the baby is at a time when the risk of intrauterine death and irreversible complications of organ function in the context of prolonged pregnancy

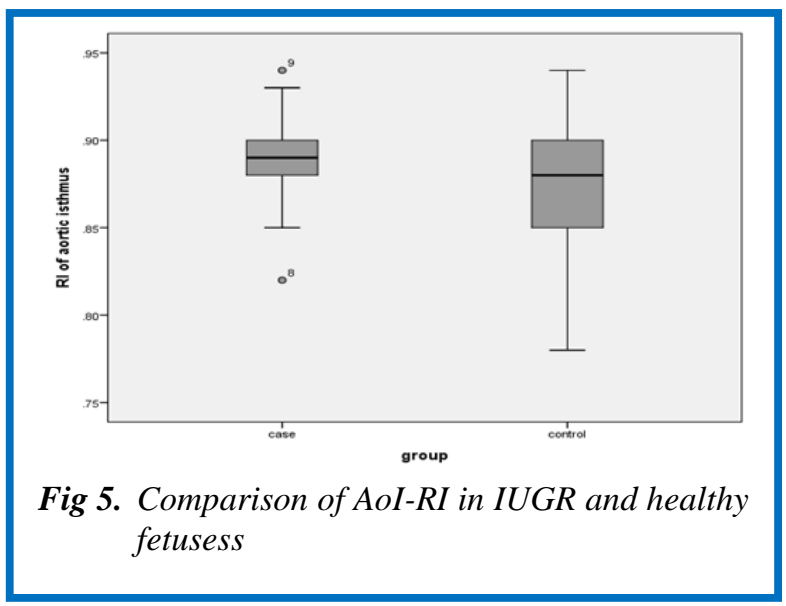

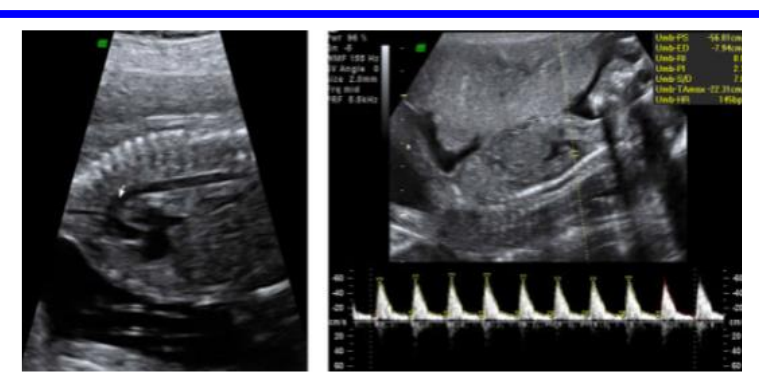

a. Arrow indicates point distal to the left subclavian artery and typical blood flow velocity waveforms.

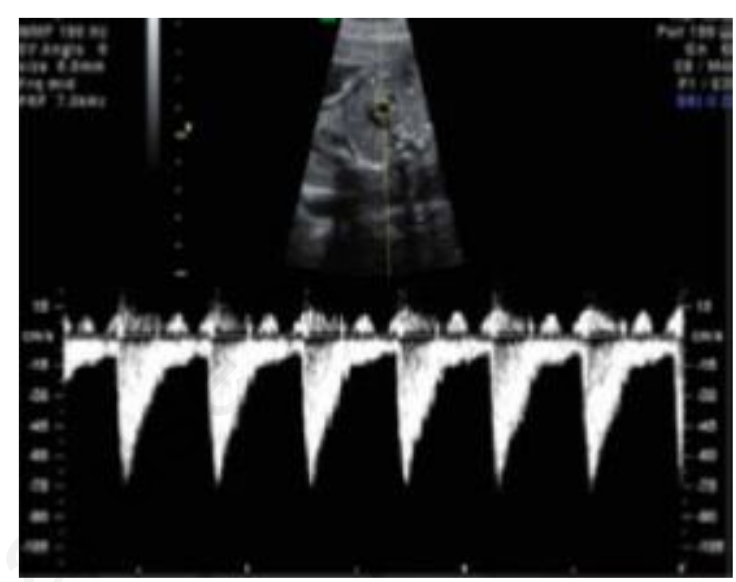

b.Three vessel views with cursor placed on AoI at point proximal to ductus arteriosus joining the descending aorta.
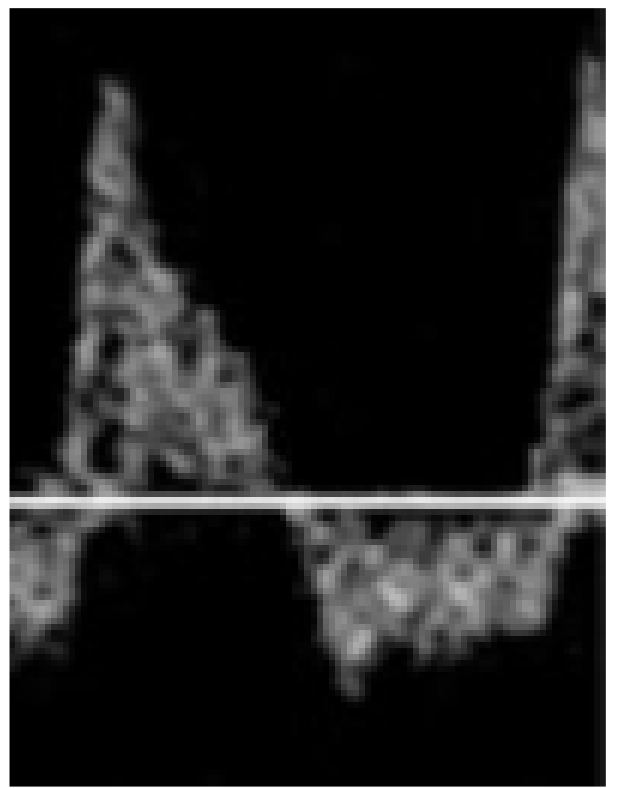

c Reversed blood flow during diastole and antegrade flow during systole.

Fig 4. The fetal aortic isthmus at week 20 of pregnancy. 
Table 1. Mean standard deviation and range of RI in both case and control groups

\begin{tabular}{|ll|l|l|l|l|l|}
\hline group & & $\mathrm{N}$ & Minimum & Maximum & Mean & Std. Deviation \\
\hline case & RI of aortic isthmus & 30 & .82 & .94 & .89 & .02 \\
& Valid N (listwise) & 30 & & & & \\
\hline control & RI of aortic isthmus & 30 & .78 & .94 & .87 & .04 \\
& Valid N (listwise) & 30 & & & & \\
\end{tabular}

is greater than the preterm birth risk. Longitudinal data show a consistent pattern of hemodynamic changes which forms the basis of surveillance and follow-up with Doppler in the IUGR. ${ }^{18-21}$ So far, several longitudinal studies have been performed on measurement of aortic isthmus in uncomplicated pregnancies, but data are still incomplete. ${ }^{21}$ A previous study indicated that peak systolic velocity (PSV) and AoI pulsatility index (PI) can be markedly elevated in AGA fetuses by increasing gestational age. Furthermore, afford mentioned study demonstrated noremarkable mean difference in measurements of AoIPI or AoI-PSV based on the intra- and interoperator variability. In addition, AoI-PI and AoI-PSV from SGA fetuses were not considerably different from those of AGA or IUGR fetuses. ${ }^{22}$ A study evaluated features and correlation with perinatal outcome of AoI circulation using Doppler method in IUGR fetuses with placental insufficiency. Adverse perinatal outcome has been found to be markedly linked to elevated AoI-PI (Retrograde flow in the AoI ). On the other hand, it has been revealed that retrograde blood flow in the AoI could be correlated with adverse perinatal outcome. Furthermore, AoI-PSV and AoI-TAMXV have been determined to be $<5$ (th) centile in $78 \%$ and $94 \%$ of subjects, respectively; where AoI-PI has been revealed to be $>95$ (th) centile in $41 \%$ of cases. Aforementioned study suggested the key role of Doppler imaging of the AoI for severe IUGR surveillance. In another study conducted in Italy in 2008, AoI-PI was measured in 70 fetuses (20 cases of IUGR and 50 normal cases) at 2436 weeks' gestation whereas all measurements were performed in both the LAA and $3 \mathrm{~V}$ views, and compared with each other. The results of mentioned study exhibited a high degree of reliability between PI values achieved from LAA and $3 \mathrm{~V}$ views in both IUGR and normal fetuses. In addition, the $3 \mathrm{~V}$ view has been remarkably recorded to be less time-consuming, when comparing with those from the LLA view, where higher intra-observer reproducibility has been reveled in this regard. ${ }^{23,24}$ In our study, we also compared RI values obtained from LAA and $3 \mathrm{~V}$ views in both IUGR and normally grown fetuses. The mean RI of aortic isthmus in the IUGR fetuses was determined as 0.89 ( $\mathrm{SD}=$ $0.02)$, comparing the highest with the lowest scores (0.82 and 0.94). The mean RI of the fetal aortic isthmus in the control group was obtained to be 0.87 ( $\mathrm{SD}=$ $0.04)$, comparing the highest with the lowest scores (0.78 and 0.94). Given Mann-Whitney test, mean RI of isthmic aorta did not exhibit a significant difference in case and control groups $(\mathrm{P}=0.08)$. Regarding to the previous studies in this area, the accepted protocol has not been achieved for the clinical surveillance of the IUGR fetuses based on the use of Doppler imaging. In this regard, longitudinal and observational studies would show more information about the natural history and pathophysiology of intrauterine growth-restricted fetuses in relation to Doppler indexes. A randomized clinical trial on the blood flow of UA and embryo in Europe (TRUFFLE study), have evaluated the fetuses' heart with a change in the latency of the DV stream using three randomized arms cardiotocography.

The data from this study and other studies provide appropriate guidance regarding the role of doppler in the timing of delivery and control of effective factors. Regarding to available evidence, the evaluation of Doppler waveforms of the AoI can be an important element in the evaluation of fetuses with the IUGR, where they have an abnormal Doppler of UA. However, prior to entering these indexes into clinical practice for preventing premature birth, more prospective studies are needed in relation to AoI indexes and other arterial Doppler indexes with perinatal outcomes. ${ }^{25,26}$

\section{List of acronyms}

AFI - amniotic fluid index

AGA - appropriate for gestational age

AoI - aortic isthmus

DV - Dactus Venus

IUGR - Intrauterine growth restriction

PI - pulsatility index

PSV - peak systolic velocity 


\section{Color Doppler sonography of the aortic isthmus in IUGR}

Eur J Transl Myol 28 (4): 370-375, 2018

RI - resistive index

SFH - symphysial fundal height

TAMXV - time-averaged maximum velocity

\section{Author's contributions}

LY,MGH, ZS, and GHA equally participated in experimental design, data collection, writing and revision of the manuscript.

\section{Acknowledgments \\ Funding: None.}

\section{Conflict of Interest}

The authors declare no conflicts of interests.

\section{Ethical Publication Statement}

We confirm that we have read the Journal's position on issues involved in ethical publication and affirm that this report is consistent with those guidelines.

\section{Corresponding Author}

Mostafa Ghadamzadeh, Department of Radiology, Hasheminejad Kidney Center (HKC), Iran University of Medical Sciences, Tehran, Iran.

Email: Mghz_61@yahoo.com

\section{E-mail of co-authors}

Ladan Younesi: younesi.l@iums.ac.ir Ghazaleh Amjad: Amjad.gh@iums.ac.ir

Zeinab Safarpour Lima: safarpourlima.z@iums.ac.ir

\section{References}

1. Owen P, Donnet ML, Ogston SA, et al. Standards for ultrasound fetal growth veloctity. Br J Obstet Gynaecol 1996;103:60-9.

2. Alexander GR. Himes JH, Kaufman RB, Mor J, Kogan M. A United States national reference for fetal growth. Obstet Gynecol 1996;87:163-8

3. Holmes RP, Holly JMP, Soothill PW. A prospective study of maternal serum insulin- like growth factor- I in pregnancies with appropriately grown or growth restricted fetuses. Br J Obstet Gynaecol 1998;105:1273-78

4. Zare Mehrjardi M, Darabi M, Bagheri SM, et al. The role of ultrasound (US) and magnetic resonance imaging (MRI) in penile fracture mapping for modified surgical repair. Int Urol Nephrol 2017;49:937-45.

5. Seeds JW. Impaired fetal growth: Definition and clinical diagnosis. Obstet Gynecol 1984;64:30310.

6. Seeds JW, Peng T. Impaired growth and risk of fetal death: Is the tenth percentile the appropriate standard? Am J Obstet Gynecol 1998;178:658-69.
7. Gardosi J, Chang A, Kalyan B, et al. Customized antenatal growth charts. Lancet 1992;339:283-7.

8. Gardosi J, Francis A. Controlled trial of fundal height measurement plotted on customized antental growth charts. $\mathrm{Br} \mathrm{J}$ Obstet Gynaecol 2000;106:309 -17.

9. Usher R, Mclean F. Intrauterine growth of liveborn cauca-sian infants at sea level: Standards obtained from measurements in 7 dimensions of infants born between 25 and 44 weeks gestation. J Pediatr 1969;74:901-10.

10. Mc Intire DD, Bloom SL, Casey BM, Leveno KJ. Birthweight in relation to morbidity and mortality among newborn infants. $\mathrm{N}$ Engl $\mathrm{J}$ Med 1999;340:1234-8

11. Snijders RJM, Nicolaides KJ. Fetal biometry at 14 to 40 week gestation. Ultrasound Obstet Gynecol 1994;4:34 -48.

12. Walraven GEL, Mkanje RJB, van Roosmalen J, et al. Single pre-delivery symphysis- fundal height measurement as a predictor of birthweight and multiple pregnancy. Br J Obstet Gynaecol 1995; 102: $525-9$

13. Jensen $\mathrm{OH}$, Larsen S. Evaluation of symphysis fundus measurements and weighing during pregnancy. Acta Obstet Gyneceol Scand 1991;70:13-6.

14. Larsen T, Larsen JF, Petersen S, Greisen G. Detection of small-for-gestation-age fetuses by ultrasound screening in a high risk population: A randomized controlled study. Br J Obstet Gynecol 1992;99:469-74.

15. Fouron JC. The unrecognized physiological and clinical significance of the fetal aortic isthmus. Ultrasound Obstet Gynecol 2003;22:441-47.

16. Barker DJ, Osmond C, Golding J, Kuh D, Wadsworth ME. Growth in utero, blood pressure in childhood and adult life, and mortality from cardiovascular diseases. BMJ 1989;289:564-7.

17. Pollack RN, Divon MY. Intrauterine growth retardation: definition, classification, and etiology. Clin Obstet Gynecol 1992;35: 99-107.

18. Baschat A, Gembrach U, Harman C. The sequence of changes in Doppler and biophysical parameters as severe fetal growth restriction worsens. Ultrasound Obstet Gynecol 2001;18: 571-7.

19. Hecher K, Bilardo CM, Stigter RH, et al. Monitoring of fetuses with intrauterine growth restriction: a longitudinal study. Ultrasound Obstet Gynecol 2001;18:564-70. 


\section{Color Doppler sonography of the aortic isthmus in IUGR}

Eur J Transl Myol 28 (4): 370-375, 2018

20. Ferrazzi E, Bozzo M, Rigano S, et al. Temporal sequence of abnormal Doppler changes in the peripheral and central circulatory systems of the severely growth-restricted fetus. Ultrasound Obstet Gynecol 2002;19:140-46.

21. Del Rio M, Martinez JM, Figueras F, et al. Reference ranges for Doppler parameters of the fetal aortic isthmus during the second half of pregnancy. Ultrasound Obstet Gynecol 2006;28:71-6

22. Kennelly MM, Farah N, Hogan J, et al. Longitudinal study of aortic isthmus Doppler in appropriately grown and small-for-gestational-age fetuses with normal and abnormal umbilical artery Doppler. Ultrasound Obstet Gynecol 2012;39:41420.

23. Del Río M, Martínez JM, Figueras F, et al. Doppler assessment of the aortic isthmus and perinatal outcome in preterm fetuses with severe intrauterine growth restriction. Ultrasound Obstet Gynecol 2008;31:41-7
24. Rizzo G, Capponi A, Vendola M, et al. Use of the 3-Vessel View to Record Doppler Velocity Waveforms From the Aortic Isthmus in Normally Grown and Growth-Restricted Fetuses Comparison With the Long Aortic Arch View. J Ultrasound Med 2008;27:1617-22.

25. Lees C, Baumgartner H. The TRUFFLE study-a collaborative publicly funded project from concept to reality: how to negotiate an ethical, administrative and funding obstacle course in the European Union. Ultrasound Obstet Gynecol 2005;25:105-7.

26. Kennelly MM, Farah N, Turner MJ, Stuart B. Aortic isthmus Doppler velocimetry: role in assessment of preterm fetal growth restriction. Prenat Diagn 2010;30:395-401.

Received for publication: 15/08/2018 Revision received: 03/09/2018

Accepted for publication: 03/09/2018 